\title{
Prevalencia del Síndrome de Burnout en docentes: Factores asociados al estatuto de vinculación laboral en Colombia
}

\author{
Jorge Iván Posada Quintero', Paula Natalia Molano Vergara ${ }^{2}$, Ronald Mauricio Parra \\ Hernández $^{3}$, Francy Yaneth Brito Osorio ${ }^{4}$ \& Edward Andrey Rubio Orozco 5
}

Recibido:29/08/2018 Aceptado: 26/02/2019

DOI: $10.21772 /$ ripo.v37n2a04

\begin{abstract}
Resumen
El presente artículo tuvo tres objetivos fundamentales: 1. analizar los principales factores que inciden en la presencia de Burnout en docentes; 2. determinar los síntomas que lo evidencian; 3. establecer bajo qué estatuto (1278 o 2277) los docentes son más susceptibles a padecer el síndrome. Los datos fueron obtenidos mediante un cuestionario $(\mathrm{n}=54)$, el cual fue convalido y calculada su fiabilidad a través de la prueba Alfa de Cronbach (Escala General ; Factores de Riesgo y Síntomas de Burnout ). Los análisis de correlación se realizaron con la prueba no paramétrica Chi cuadrado; para hallar diferencias entre las variables se usó la U de Mann Whitney. Los resultados del análisis evidenciaron que los principales problemas relacionados con síntomas de Burnout en docentes fueron: trabajar horas extras, falta de espacios de comunicación con los jefes, salario inadecuado para satisfacer sus necesidades y falta de apoyo de los jefes frente a dificultades con padres de familia. Finalmente, se concluyó que los docentes regulados con el estatuto 1278 son más susceptibles al síndrome en razón de presentar unas condiciones laborales menos adecuadas que los regulados por el estatuto 2277
\end{abstract}

Palabras clave: Burnout, docentes, estatutos, prevalencia, síntomas, Riesgo Laboral, Colombia.

\section{Prevalence of Burnout Syndrome in teachers: Factors associated with the statute of employment in Colombia}

\begin{abstract}
The present article has three objectives: 1 . To analyze the main factors that affect the presence of Burnout in teachers; 2 . to determine the symptoms that show it; 3 . to establish under which statute (1278 or 2277) the teachers are more susceptible to suffer the syndrome. The data were obtained by means of a questionnaire $(n=54)$, which was validated through the Cronbach's Alpha test (General Scale $\alpha=0.984$; Risk factors $\alpha=.96$ and Symptoms of Burnout $\alpha=.977$ ). The correlation analysis were performed with the non-parametric Chi square test; to find the differences between the variables the Mann Whitney $U$ test was used. The results of the analysis showed the main problems related to the Burnout factors in teachers were: working overtime, lack of communication spaces with the bosses, inadequate salary to meet their needs and lack of support from the bosses. Finally, it is concluded that teachers regulated by statute 1278 were more susceptible to the syndrome because of the lower working conditions.
\end{abstract}

Keywords: Burnout, incidence, symptoms, Labor Risks, teachers, statutes, Colombia

\footnotetext{
1 Ms. en Educación. Candidato a Doctor en Ciencias de la Educación. Institución Educativa Técnica Niña María. E-mail: jorgefresno86@gmail.com

2 Ms. en Educación. Corporación universitaria Minuto de Dios. E-mail: paulanmolano90@hotmail.com

3 Ms. en Educación. Estudiante de Doctorado. Investigador Grupo GIEPETO. E-mail: orniparra@yahoo.es

4 Ms. en Educación con Mención en Pedagogía. Corporación universitaria Minuto de Dios - UNIMINUTO. E-mail: fyboearo@hotmail.com 5 Ms. en Enseñanza de las Ciencias Exactas y Naturales. Estudiante De Doctorado. Docente Lider De Semillero De Investigacion. Corporación Universitaria Minuto De Dios. E-mail: earofybo@hotmail.com

Cómo citar: Posada Quintero, J. I.; Molano Vergara, P. N.; Parra Hernández, R. M.; Brito Osorio, F. Y. \& Rubio Orozco, E. A. (2019). Prevalencia del Síndrome de Burnout en docentes: Factores asociados al estatuto de vinculación laboral en Colombia. Revista Interamericana de Psicología Ocupacupacional, 37-2, 119-133. DOI: 10.21772/ripo.v37n2a04
} 


\section{Introducción}

Los Factores Psicosociales (FP) asociados al trabajo han sido ampliamente estudiados por la comunidad académica, principalmente desde finales de la década pasada (Sauter, Murphy Hurrell \& Levi, 2001). Toda persona, en ejercicio de su labor profesional, enfrenta tensiones psicológicas, sociales, locativas y ambientales relacionadas con estos factores. Desde la perspectiva de la salud laboral, tales problemas no solo pueden afectar a los trabajadores en su vida profesional, sino también familiar y de manera directa a las organizaciones en las cuales se desempeñan. En los últimos 10 años los FP se han vuelto más frecuentes, conocidos y diagnosticados, tomando importancia a tal grado que la Organización Mundial para la Salud (OMS) los ha catalogado como de riesgo laboral (Gutiérrez, Celis, Moreno, Farías, \& Suárez, 2006). La Agencia Europea de Seguridad y Salud, en un trabajo desarrollado en el 2000, mencionó la necesidad de investigar y establecer estos nuevos riesgos en la salud (León, Matsui, Aranda \& León, 2008). Así mismo, los reportes de investigación en el campo de la seguridad y salud en el trabajo, manifiestan que hay relaciones causales directas entre un desajuste por las exigencias personales y laborales congruentes con el estrés laboral y que causan efectos crónicos en la salud (Porras, 2017).

En la actualidad son conocidos diversos síndromes relacionados con actividades de índole laboral como: Tecnoestrés, Cronos, Anát, Mobbing, Ganímides, Burnout, entre otros (Medina, 2015; Aragüez, 2017). No obstante, éste artículo sólo se centra en Burnout, debido a que su estudio puede contribuir a desarrollar intervenciones apropiadas para corregirlo, bien sea de tipo individual, acentuando la acción psicológica, o bien de tipo social u organizacional, incidiendo en las condiciones de trabajo (Pérez, 2010). Además, existe un consenso básico sobre la importancia de diagnosticar el síndrome $\mathrm{y}$ promover programas de actuación.
Gutiérrez et al. (2006) señalan que las consecuencias fisiológicas profundas del estrés fueron demostradas por primera vez de manera empírica por Hans Selye en 1936, quien describió un síndrome producido por diversos agentes nocivos. Investigaciones subsecuentes señalaron un amplio rango de efectos fisiológicos adversos del estrés tanto en animales como en humanos. Así mismo, Hans Selye describió tres etapas del estrés: alarma o reacción, adaptación y agotamiento o descompensación. En esta última etapa se produce una pérdida de recursos adaptativos que va acompañada de un grupo típico de síntomas de ansiedad (Palmer, Gómez, Cabrera, Prince \& Searcy, 2005).

Sin embargo, el síndrome de Burnout no fue descrito hasta 1974, cuando el psiquiatra Herbert J. Freudenberger, quien trabaja en una clínica para toxicómanos en Nueva York, observó que la mayoría de los voluntarios que atendían drogadictos después de un año, sufrían pérdida progresiva de energía que los conducía hasta el agotamiento y provocaba síntomas de ansiedad y depresión, así como desmotivación en el trabajo y agresividad con los pacientes (Gutiérrez et al., 2006; López, 2004). A raíz de estos hallazgos explicó que el Burnout es fallar, desgastarse o sentirse exhausto debido a las demandas excesivas de energía, fuerza espiritual o recursos.

En ese mismo año, la psicóloga social Cristina Maslach, en estudios de respuestas emocionales de profesionales, consideró que estos eran afectados por "Sobrecarga emocional", síndrome de Burnout o síndrome del quemado, "señalando que éste hace referencia a un agotamiento emocional, despersonalización y baja realización personal, que se da en sujetos que trabajan con personas" (Maslach \& Jackson, 1981, p. 1). De igual manera, otros autores lo conceptualizan como el síndrome del desgaste profesional y de fatiga en el trabajo (Guevara, Henao \& Herrera, 2004; Gutiérrez et al., 2006; López, 2004; Méndez, 2004). 
En 1978, Pines y Kafry definieron Burnout "como una experiencia que genera agotamiento físico, emocional y actitudinal" (Ortega \& Giménez, 2013, p. 5). Maslach y Jackson en 1981, consideraron que el síndrome se produce por una forma inadecuada de afrontar un estrés emocional crónico cuyos rasgos principales son el agotamiento emocional, la despersonalización y la disminución del desempeño personal (López, 2004). Maslach (1982) afirmó que las personas afectadas por el Burnout suelen perder tolerancia a la frustración, aumentar la irritabilidad y desarrollar una experiencia especialmente sensible a los fallos organizacionales y a las relaciones interpersonales.

Moriana y Herruzo (2004), determinaron que a nivel mundial los estudios descriptivos con diseños correlacionales que analizan la relación entre estrés y Burnout con otras variables fundamentalmente sociodemográficas, se repiten en casi todos los países. En Alemania, KnightWegenstein (1973) realizó uno de los estudios pioneros en el ámbito de la educación en el que encontró que un 87,6\% de 9129 profesores parecían afectados por un elevado grado de tensión en el trabajo. Así mismo, Moriana y Herruzo (2004) postularon que "en esta línea, se repite este mismo tipo de investigaciones en países de todo el mundo, señalando elevadas puntuaciones en diversas medidas de estrés y Burnout" (p. 599).

Aunque diversos autores en principio consideraron que el Burnout era un síndrome exclusivo de profesionales en servicios sociales, o que trabajan en contacto directo con personas a las que se prestan servicios, hoy en día se considera que esté puede presentarse en trabajos que no exigen dicho contacto (Rojas, Zapata \& Grisales, 2009). En general se conocen diversos estudios que han evidenciado el síndrome de Burnout en distintas profesiones: administrativos y auxiliares administrativos, enfermeros, deportistas, monitores de educación especial, trabajadores de centros ocupacionales y sociales, vigilantes de instituciones penitenciarias, voluntarios $\mathrm{y}$ docentes (Ortega y López, 2004; Rojas, Zapata \& Grisales, 2009; Saquero, Jaime \& Ortín. 2018).

En el ámbito educativo Díaz, López \& Varela (2010) manifestaron que en diversos estudios los docentes mostraron una alta vulnerabilidad al Burnout, debido a la realización simultánea de múltiples actividades y funciones que implican asegurar que los estudiantes reciban una educación de calidad, así como exigencias grandes y conflictivas, no solo por parte de los alumnos, sino también de las familias, redes sociales y la administración (Aldrete, Pando, Aranda \& Balcázar, 2003; Ponce, Bulnes, Aliaga, Atalaya \& Huertas, 2005; Redó, 2017).

De igual manera, se considera que este síndrome se está convirtiendo en un serio problema no sólo para el colectivo docente, sino también para el sistema educativo en general, por sus consecuencias directas sobre la calidad de la enseñanza y su correspondiente efecto negativo creciente en bajas laborales, rotación, absentismo y decrecimiento en la productividad de la enseñanza (Doménech, 1995; Extremera, Fernández-Berrocal \& Durán, 2003; Redó, 2017). León et al. (2008) concluyen que la salud mental de los docentes es un problema actual que debe ser considerado y que puede estar asociado al síndrome de Burnout.

Entre los diversos estudios, se puede mencionar el de Aldrete et al. (2003) en docentes de educación básica primaria en Guadalajara (México, 2003), quienes encontraron altos niveles de cansancio emocional $(25,9 \%)$, baja realización personal en el trabajo $(21,6 \%)$ y despersonalización (5,6\%). Así mismo, en el ámbito nacional colombiano, se han desarrollado diversas investigaciones que destacan la prevalencia del síndrome de Burnout en docentes (Viloria \& Pareces, 2002; Hermosa, 2006; Restrepo, Colorado \& Cabrera, 2006; Yépez y Soracá, 2006; Rojas, Zapata, \& Grisales, 2009; Padilla et al., 2009; Correa-Correa, Muñoz-Zambrano \& Chaparro, 2010), muchas de las cuales estudian 
la incidencia del Síndrome mediante el uso del Maslach Burnout Inventory (MBI).

Entre los estudios realizados en Colombia, se destaca el de Padilla, et al. (2009) el cual halla una prevalencia menor del síndrome en docentes mayores de 55 años, con 21 a 30 años de experiencia, en aquellos que trabajan solamente una jornada y los que tienen cargos directivos. Así mismo vale enunciar el de Díaz et al. (2010) que resalta la relación del síndrome con el Estrés de Rol y los factores organizacionales de supervisión, preocupaciones profesionales y el nivel escolar en el que imparten clase los docentes.

En general, las investigaciones a nivel nacional y mundial han demostrado la presencia del síndrome de Burnout en docentes, analizando factores causales y grado de prevalencia. No obstante, se desconocen los trabajos que comparen el grado de prevalencia de Burnout en docentes regidos bajo estatutos de vinculación laboral 1278 frente al grupo de docentes regidos por el estatuto 2277.

En el caso de Colombia la profesión docente está reglamentada por dos estatutos (1278 y 2277), cada uno de los cuales esta específicamente estructurado según el ejercicio de funciones, cumplimiento de requisitos como ingreso, permanencia y ascenso. Para el caso del nuevo estatuto (1278), el nombramiento es por méritos y contempla el desarrollo de un concurso docente, el cual implica alcanzar unos mínimos puntajes y competir con otros postulantes para el ingreso a la planta magisterial, a diferencia del antiguo estatuto que es por nombramiento directo. A nivel general se han "establecido nuevas reglas de formación, ingreso, evaluación, promoción y permanencia en la profesión, las cuales buscan incidir en los resultados de los estudiantes mediante el mejoramiento de la calidad docente" (Macia, 2009; p. 3).

Con relación a la permanencia, el 2277 es por derecho y el 1278 por idoneidad, sumado a funciones y tareas relacionadas con la evaluación docente, cuestión que no se contemplaba en el anterior estatuto. Dentro del aspecto de la remuneración, incentivos, escalafón y especialización las funciones se convirtieron en una de las maneras de cualificar y calificar la labor docente a partir de evaluación de desempeños y competencias que sólo son presentadas por los del 1278 (Cifuentes, 2013). En muchos casos es evidente que los docentes exterioricen desmotivación por no poder ascender en el escalafón docente y por ende en su remuneración salarial, pese a un sin número de evaluaciones a las que se ven sometidos de manera permanente.

Con relación a lo anterior, al analizar algunos efectos del estatuto sobre la función docente, el funcionamiento simultáneo de los decretos, incide radicalmente en la calidad de la educación, pero esto no garantiza mejorar la calidad de vida ni las condiciones laborales de los docentes. Macia (2009) señala que la profesionalización docente continúa acompañada de una heterogeneidad y desigualdad creciente en términos de género, formación profesional, condiciones laborales y salariales.

De manera que este estudio busca determinar la prevalencia de Burnout en la Institución Educativa Técnica San José de Fresno.

\section{Metodología}

En este estudio se analizó la prevalencia del Síndrome de Burnout en docentes y la presencia de factores de riesgo asociados al estatuto de vinculación laboral. Para ello se ejecutó un marco metodológico basado en cinco (5) componentes: participantes, diseño, procedimiento, instrumentos de medida y análisis de datos. La investigación corresponde a un estudio de tipo correlacional descriptivo (Campoy, 2018). La muestra estuvo conformada por docentes de la Institución Educativa Técnica San José de Fresno (Tolima). La información se obtuvo a partir de una encuesta aplicada a 
la totalidad de los docentes (54), con edades comprendidas entre los 22 y 63 años $(D E=12)$. De los cuales 43 (79.63\%) estaban nombrados en propiedad y $11(20.37 \%)$ provisionales; el mayor porcentaje de encuestados correspondió a mujeres (76\%). Para efectos del análisis, la muestra fue divida en dos grupos: docentes vinculados bajo el decreto $2277 / 79(\mathrm{~N}=17 ; 31.5 \%$, ) y $1278 / 02$ $(\mathrm{N}=37 ; 68.5 \%$; ).

Inicialmente se solicitaron las características sociodemográficas (estatuto de vinculación laboral, edad y sexo), seguidamente se indagó por 26 ítems correspondientes a 13 factores y 13 síntomas que evidencian el síndrome. Los factores estudiados fueron: comunicación con superiores, salario suficiente, trabajar horas extras, reconocimiento social, faltas y sanciones, apoyo de superiores, pausas durante la jornada, valoración del trabajo, tareas acordes a la profesión docente, relación con sus compañeros, horario laboral, afiliación sindical y oportunidades de capacitación. Los síntomas analizados a través del instrumento fueron: pérdida del apetito, dificultad de comunicación, dolor de cabeza, problemas gástricos, sensación de depresión, consulta médica por lesiones, desesperación, sensación de fatiga, consulta por salud mental, sueño irregular, sensación de tristeza, irritabilidad y consulta por lesiones de voz. Estas variables fueron tomadas a partir de las especificaciones de la Batería de instrumentos para la Evaluación de factores de riesgos psicosocial del Ministerio de la Protección social (2010).

El instrumento se validó y calculó su fiabilidad a través de la prueba Alpha de Cronbach tanto en la escala general (), como en las sub-escalas de Factores de riesgo () y Síntomas de Burnout (). Estos valores fueron significativamente altos y representan una alta consistencia interna del instrumento aplicado y una sólida validez de constructo. Para lo cual, se usó el Paquete Estadístico para Ciencias Sociales: IBM SPSS Statistics 22, así como en el análisis de los datos.
Para establecer los principales factores y síntomas de Burnout en ambos decretos de profesionalización se utilizaron gráficas de contingencia que permitieron evidenciar la presencia del síndrome en ambos estatutos. Se establecieron cuatro categorías de medida, de la siguiente manera: Nunca (0), casi nunca (1), Algunas veces (2) y siempre (3). En segunda instancia se comparó la prevalencia de los factores de riesgo y síntomas del síndrome entre ambos estatutos (1278 y 2277) acumulando las frecuencias (FA) de las cuatro categorías establecidas, seguidamente se construyeron variables categóricas que contienen esta agrupación en porcentajes, así: Baja $(0 \%<x<20 \%)$, Media $(20 \%<x<60 \%)$, Moderada $(60 \%<x<80 \%)$ y Alta $(80 \%<x<100 \%)$, tal como se explica en la formula a continuación:

Frecuencia acumulada $(\mathrm{FA})=\frac{\sum \text { Categoría parciales }}{\text { Total de factores o síntomas }} \times 100$

Así mismo, se compararon las frecuencias acumuladas (FA) de los factores que causan Burnout y los síntomas que lo evidencian, entre ambos estatutos usando como medida de la prueba Chi-Cuadrado, a partir de las variables contrastadas en tablas de contingencia, para analizar su comportamiento en cada uno de los grupos estudiados, lo que permitió evidenciar qué factores diferían en mayor medida. Para aceptar o rechazar la diferencia estadística entre los grupos se usó la prueba U de Mann Withney, debido al carácter no paramétrico y la distribución no normalizada encontrados en las variables del estudio.

\section{Resultados}

En la Tabla 1 se presentan los estadísticos básicos para cada una de las variables del estudio; la media, desviación estándar, correlación total de elementos corregidos y Alpha de Cronbach si el elemento es suprimido. 
Tabla 1. Variables del estudio, media $(\mu)$, desviación estándar (, correlaciones y fiabilidad.

\begin{tabular}{|c|c|c|c|c|}
\hline Variables del estudio & $\mu$ & & Correlación & $\begin{array}{c}\text { de Cronbach si se elimina } \\
\text { elemento }\end{array}$ \\
\hline Tareas Acordes & 2.69 & .639 & .860 & .983 \\
\hline Comunicación con Jefes & 2.17 & .637 & .715 & .984 \\
\hline Relación con sus Compañeros & 2.63 & .487 & .766 & .984 \\
\hline Pausas durante la jornada & 1.65 & 1.246 & .915 & .983 \\
\hline Sensación de Fatiga & 2.33 & .673 & .819 & .983 \\
\hline Apoyo de los Jefes & 2.39 & .596 & .776 & .983 \\
\hline Dolor de Cabeza & 1.59 & .836 & .830 & .983 \\
\hline Irritabilidad & 1.57 & .924 & .946 & .982 \\
\hline Sensación de tristeza & 1.04 & 1.063 & .922 & .982 \\
\hline Pérdida del Apetito & 1.04 & 1.243 & .868 & .983 \\
\hline Sueño Irregular & 1.39 & .878 & .913 & .982 \\
\hline Depresión & 1.30 & 1.127 & .940 & .982 \\
\hline Desesperación & 1.37 & .875 & .912 & .982 \\
\hline Dificultad de Comunicación & .98 & .835 & .856 & .983 \\
\hline Consulta por Salud Mental & .56 & 1.144 & .692 & .984 \\
\hline Consulta por Lesiones Músculo-Esqueléticas & 1.07 & 1.147 & .918 & .982 \\
\hline Consulta por Lesiones de Voz & 1.04 & 1.063 & .902 & .982 \\
\hline Consulta por Problemas Gástricos & 1.26 & 1.013 & .857 & .983 \\
\hline Oportunidades de Capacitación & 1.67 & .911 & .889 & .983 \\
\hline Salario Suficiente & 2.00 & .932 & .804 & .983 \\
\hline Valoración del Trabajo realizado & 1.89 & .816 & .842 & .983 \\
\hline Oportunidades de Afiliación Sindical & 2.83 & .637 & .549 & .984 \\
\hline Faltas y Sanciones por parte de jefes & 1.26 & 1.152 & .891 & .983 \\
\hline Horario Laboral & 2.65 & .828 & .704 & .984 \\
\hline Trabajo de Horas Extras & 1.69 & 1.043 & .870 & .983 \\
\hline Reconocimiento Social & 1.65 & 1.246 & .921 & .982 \\
\hline
\end{tabular}

Los valores positivos y significativamente altos en las correlaciones indican una alta consistencia interna del instrumento utilizado y un aporte sustancial de cada ítem al estudio de la prevalencia del síndrome de Burnout, validando
Por otro lado, teniendo en cuenta el índice general Alpha de Cronbach (), en la quinta columna se verifica que la fiabilidad no aumenta con la eliminación de ninguno de los ítems, corroborando su pertinencia dentro de la escala.

La composición de la muestra correspondió a $39(72 \%)$ personas con pareja habitual estable, $43(79.63 \%)$ docentes nombrados en propiedad y $11(20.37 \%)$ provisionales. 
así el modelo utilizado en la recolección de la información.

Tabla 2. Prevalencia del síndrome de Burnout en docentes de I. E. Técnica San José de Fresno.

\begin{tabular}{lcccc}
\hline \multirow{2}{*}{$\begin{array}{c}\text { Prevalencia de } \\
\text { Burnout }\end{array}$} & \multicolumn{4}{c}{ Decreto de profesionalización } \\
\cline { 2 - 5 } \multicolumn{1}{c}{ Grado } & \multicolumn{2}{c}{$\mathbf{1 2 7 8 / 0 2}$} & \multicolumn{2}{c}{$\mathbf{2 2 7 7 / 7 9}$} \\
\hline Recuento & \% & Recuento & \% \\
\hline Meja (0 al 20\%) & 9 & 24.3 & 5 & 29.4 \\
Alta (60 a 80\%) & 17 & 45.9 & 7 & 41.2 \\
Total & 11 & 29.7 & 5 & 29.4 \\
\hline
\end{tabular}

Se determinó que el $24.3 \%$ de los docentes 1278 y el $29.4 \%$ del 2277 evidencian una baja prevalencia del síndrome de Burnout, el 45.9\% y $41.2 \%$ de los docentes 1278 y 2277 respectivamente presentan una prevalencia media, mientras que el $29.7 \%$ y 29,4 de los decretos 1278 y 2277 tienen una prevalencia alta respectivamente (Tabla 2). Los resultados señalaron que los tres principales síntomas que evidenciaron el síndrome de Burnout en docentes de la Institución Educativa fueron: presentar fatiga al terminar la jornada laboral, sueño irregular y depresión.

En cuanto al grado de fatiga se encontró un mayor porcentaje en docentes regulados por el decreto 1278 frente a los regulados por el decreto 2277 con un 97.2 y un $88.2 \%$ respectivamente. De igual manera, se halló que las personas reguladas por el decreto 1278 presentan sueño regular siempre (5.41\%) y en algunas oportunidades $(54.1 \%)$, en mayor porcentaje que los regulados por el decreto 2277 (52.84\%; 0\%). Así mismo, algunos docentes regulados por el decreto 1278 consideraron que se deprimen siempre $(8.57 \%)$ mientras que ninguno de los regulados por el decreto 2277 señalaron deprimirse siempre $(0 \%)$, sin embargo en mayor medida los regulados por el decreto 2277 frente a los regulados por el decreto 1278 manifiestan deprimirse algunas veces $(28.57 \%$ frente $43.75 \%)$. Estos resultados demuestran que los docentes del estatuto 1278 tienen mayor susceptibilidad de presentar el síndrome que los 2277 en la Institución Educativa, tal como lo muestra la figura 1.

Los cuatro principales problemas más relacionados con factores de Burnout en docentes de ambos estatutos fueron: trabajar horas extras, la falta de espacios de comunicación con los jefes, un salario adecuado para satisfacer sus necesidades y falta de apoyo de los jefes frente a dificultades con padres de familia.
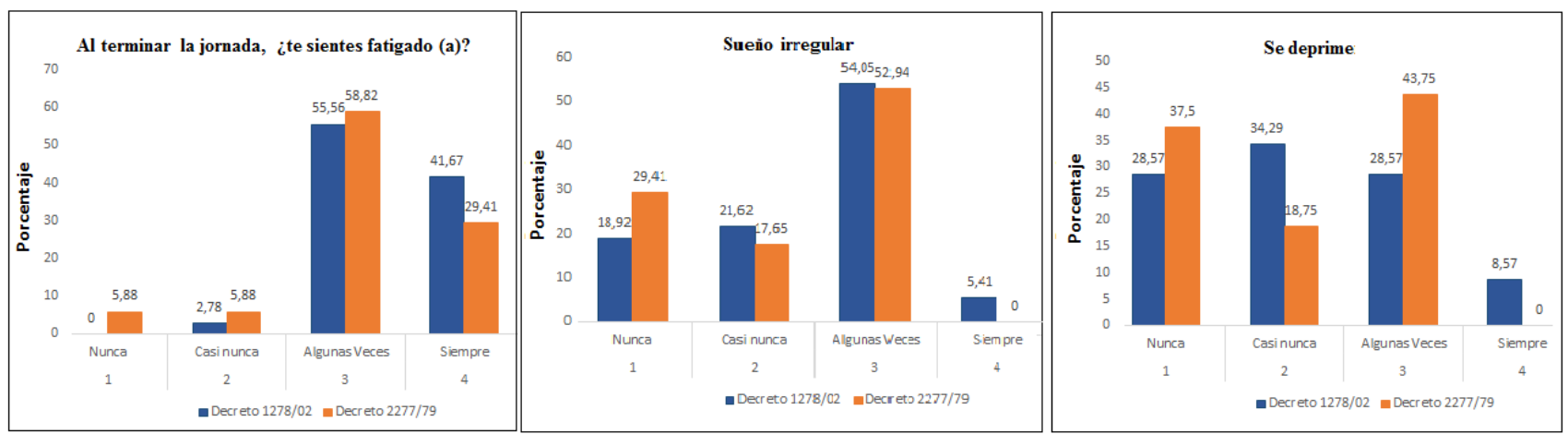

Figura 1. Principales síntomas que evidencian el síndrome Burnout en docentes de los decretos 1278 y 2277 de la I. E. Técnica San José, de Fresno. Izquierda; porcentaje de afectación por fatiga, centro; porcentaje de sueño irregular en docentes y derecha; porcentaje de docentes que se deprimen. 
De estos factores se halló que entre ambos estatutos habían diferencias en que: docentes 1278 tienen que trabajar más horas extras que los 2277 con $77.15 \%$ y $52.95 \%$ respectivamente; mientras que los canales y espacios de comunicación con los jefes son buenos en mayor porcentaje en docentes 2277 que en 1278 (algunas veces $75.68 \%$ en 1278 y $35.29 \%$ 2277; siempre con $13.51 \%$ en 1278 y $58.82 \%$ en 2277); de igual manera hubo un mayor porcentaje en docentes 1278 , en los ítem: salario no les permite satisfacer sus necesidades y ante la dificultad con padres de familia los del estatuto 2277 se sintieron más apoyados por sus jefes que los 1278 (algunas veces $56.76 \%$ frente $35.14 \%$; $35.29 \%$ frente $64.71 \%$ ) (Figura 2).
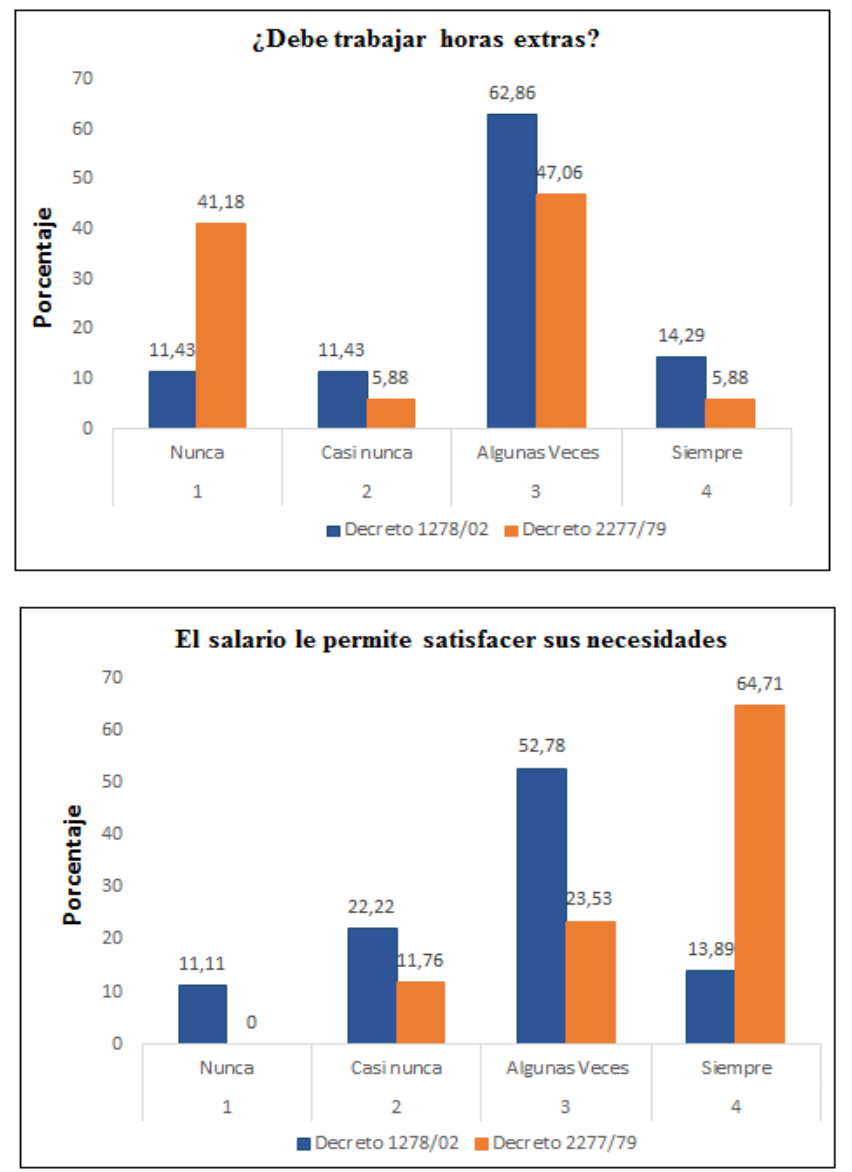

Entre los trece factores y síntomas evaluados a través de la prueba de diferencia de medias, Chi-Cuadrado (), con relación a los grupos de docentes regidos por el decreto $1278 \mathrm{y}$ 2277 los factores que variaron significativamente fueron: comunicación con superiores $(=15.64$; $p=.001)$ y salario suficiente para satisfacer sus necesidades básicas $(=14.712 ; p=.002)$ (Tabla $3)$. Mientras los síntomas que presentaron mayor diferencia fueron: dificultad en la comunicación ( $=7.118 ; p=.028)$ y pérdida del apetito $(=7.237$; $p=.065)$ (Tabla 3).
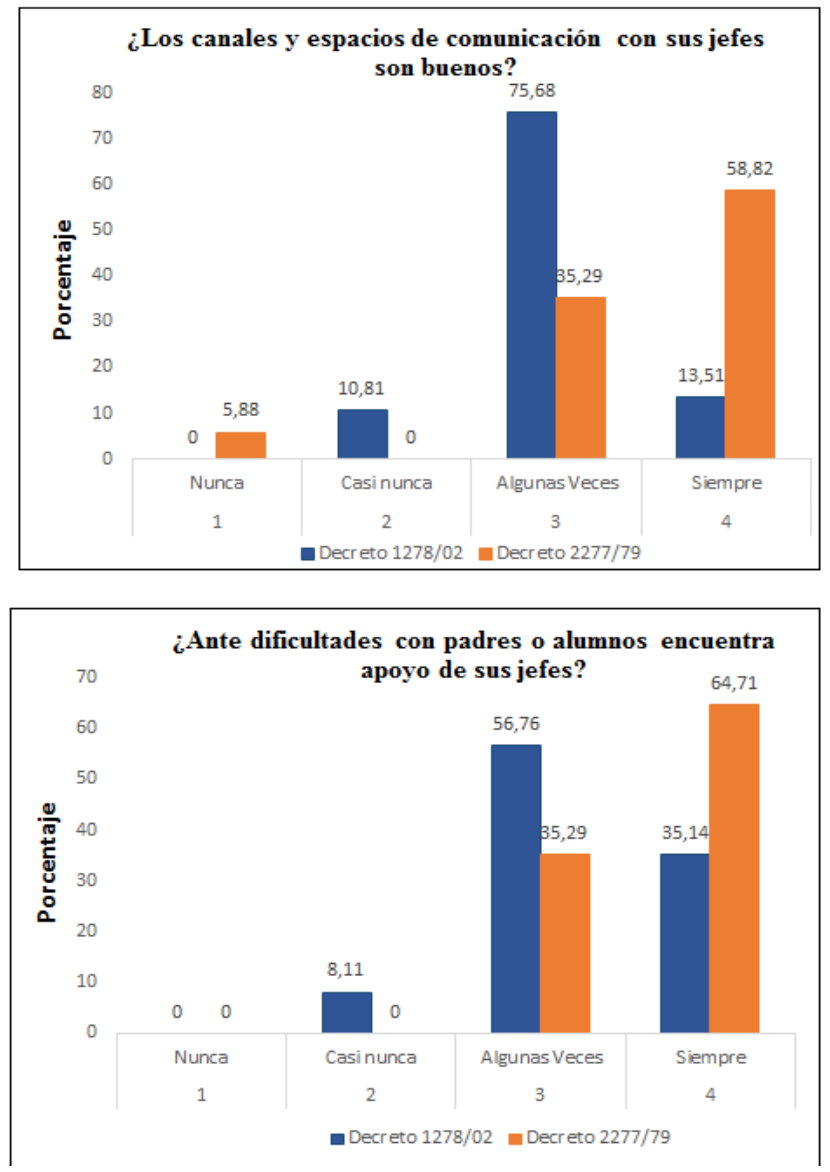

Figura 2. Principales factores que inciden en el síndrome Burnout en docentes 1278 y 2277 de la I. E. Técnica San José, de Fresno. Superior izquierda porcentaje de docentes que deben trabajar horas extras; derecha superior porcentaje de docentes que presentan canales y espacios de comunicación buenos con sus jefes e inferior izquierda porcentaje de los docentes que satisfacen sus necesidades con los salarios y derecha inferior porcentaje de docentes que tienen dificultad con padres de familia. 
Tabla 3. Comparación entre los factores y síntomas de Burnout entre docentes 1278 y 2277.

\begin{tabular}{|c|c|c|c|c|c|c|c|}
\hline Factores & Chi & gl & SSig. & Síntomas & Chi & gl & Sig. \\
\hline Comunicación con superiores & 15.64 & 3 & .001 & Dificultad de comunicación & 7.118 & 2 & .028 \\
\hline Salario suficiente & 14.712 & 3 & .002 & Pérdida del apetito & 7.237 & 3 & .065 \\
\hline Horas extras & 6.348 & 3 & .096 & Dolores de cabeza & 4.756 & 3 & .191 \\
\hline Reconocimiento social & 5.562 & 3 & .135 & Problemas gástricos & 4.745 & 3 & .191 \\
\hline Faltas y sanciones & 4.937 & 3 & .176 & Depresión & 3.31 & 3 & .346 \\
\hline Apoyo de superiores & 4.743 & 2 & .093 & Consulta por lesiones & 3.283 & 3 & .35 \\
\hline Pausas durante la jornada & 3.876 & 3 & .275 & Desesperación & 2.95 & 3 & .399 \\
\hline Valoración del trabajo & 3.178 & 3 & .365 & Sensación de fatiga & 2.894 & 3 & .408 \\
\hline Tareas acordes & 2.327 & 2 & .312 & Consulta por salud mental & 2.674 & 3 & .445 \\
\hline Relación con compañeros & 1.941 & 1 & .164 & Sueño irregular & 1.589 & 3 & .662 \\
\hline Horario laboral & 1.395 & 3 & .707 & Tristeza & 1.335 & 3 & .721 \\
\hline Afiliación sindical & 0.387 & 2 & .824 & Irritabilidad & 0.165 & 3 & .983 \\
\hline Oportunidades de capacitación & 0.078 & 3 & .994 & Consulta por lesiones de voz & 0.091 & 3 & .993 \\
\hline
\end{tabular}

Teniendo en cuenta lo anterior, se analizó la prevalencia de factores de riesgo y síntomas de Burnout entre ambos estatutos (1278 y 2277), tal como se señaló en la metodología.

Tabla 4. Prevalencia de Factores de Riesgo de Burnout de acuerdo al decreto de profesionalización.

\begin{tabular}{lcccc}
\hline \multirow{2}{*}{$\begin{array}{c}\text { Prevalencia Factores } \\
\text { de Riesgo }\end{array}$} & \multicolumn{3}{c}{ Decreto de profesionalización } \\
\cline { 2 - 5 } Grado & \multicolumn{2}{c}{$\mathbf{1 2 7 8 / 0 2}$} & \multicolumn{2}{c}{$\mathbf{2 2 7 7 / 7 9}$} \\
\hline Recuento & \% & Recuento & \% \\
\hline Baja (0 al 20\%) & 5 & 13.5 & 11 & 29.6 \\
Media (20 a 60\%) & 28 & 75.7 & 5 & 61.1 \\
Tlta (60 a 80\%) & 4 & 20.8 & 1 & 9.3 \\
Total & 37 & 100 & 17 & 100 \\
\hline
\end{tabular}

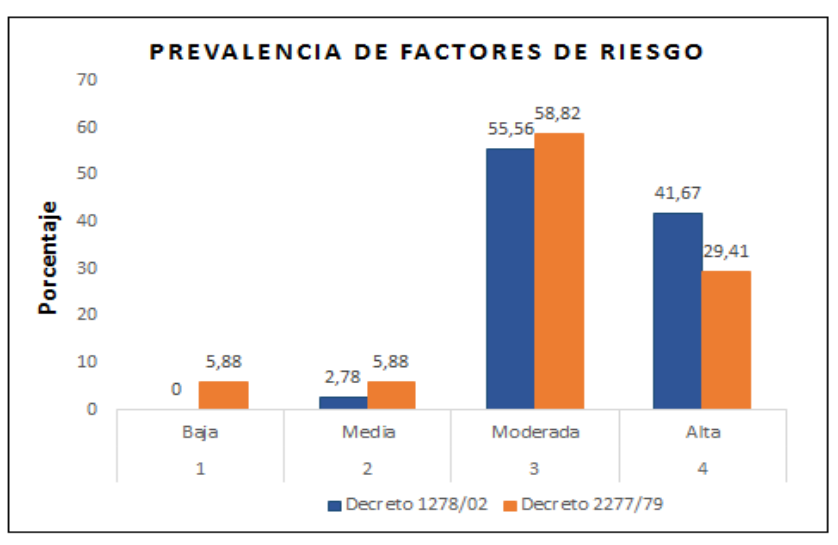

Figura 3. Principales factores que inciden en el síndrome Burnout en docentes de los decretos 1278 y 2277 de la I. E. Técnica San José, de Fresno (Tolima).
Los porcentajes observados en esta tabla revelan una diferencia entre ambos grupos frente a la prevalencia de factores de riesgo (Tabla 4). Esta diferencia se corroboró a través del índice U de Mann Whitney (Tabla 5).

Tabla 5. Diferencias significativas entre los factores de riesgo a través de U de Mann Whitney.

\begin{tabular}{lc}
\hline Estadísticos de prueba & Prevalencia Factores de Riesgo \\
\hline U de Mann-Whitney & 157.500 \\
W de Wilcoxon & 310.500 \\
Z & -3.387 \\
Sig. asintótica (bilateral) & .001 \\
\hline
\end{tabular}

La tabla 5, muestra un valor de 157.5 para el índice $\mathrm{U}$ de Mann-Whitney con un $p=$ $.001(0.1 \%$ de error) lo que permite concluir que hay evidencia estadística suficiente para demostrar que los docentes 1278 presentan una diferencia significativa frente a los docentes 2277 con relación en factores de riesgo de Burnout, producto de una mala comunicación con los jefes y salario insuficiente, esto se observa en la figura 3.

En este caso los porcentajes observados en cada columna varían de una categoría a otra entre los grupos estudiados, resaltando una presencia Alta de Burnout en $3(8.1 \%)$ docentes del 1278 frente a 0 en docentes 2277 en prevalencia de los síntomas (Tabla 6). 
Tabla 6. Síntomas de Riesgo de Burnout según el decreto de profesionalización (1278 y 2277).

\begin{tabular}{|c|c|c|c|c|c|c|}
\hline \multirow{3}{*}{$\begin{array}{c}\begin{array}{c}\text { Prevalencia de síntomas } \\
\text { Burnout }\end{array} \\
\text { Grado }\end{array}$} & \multicolumn{4}{|c|}{ Decreto de profesionalización } & & \\
\hline & \multicolumn{2}{|c|}{$1278 / 02$} & \multicolumn{2}{|c|}{$2277 / 79$} & \multicolumn{2}{|c|}{ Total } \\
\hline & Recuento & $\%$ & Recuento & $\%$ & Recuento & $\%$ \\
\hline Baja & 14 & 37.8 & 11 & 64.7 & 25 & 46.3 \\
\hline Media & 15 & 40.5 & 2 & 11.8 & 17 & 31.5 \\
\hline Moderada & 5 & 13.5 & 4 & 23.5 & 9 & 16.7 \\
\hline Alta & 3 & 8.1 & 0 & 0 & 3 & 5.6 \\
\hline Total & 37 & 100 & 17 & 100 & 54 & 100 \\
\hline
\end{tabular}

Sin embargo, el cálculo del índice U de Mann Whitney presentó lo siguiente (Tabla 7):

Tabla 7. Diferencias significativas entre los sintomas de los factores de riesgo a través de U de Mann Whitney

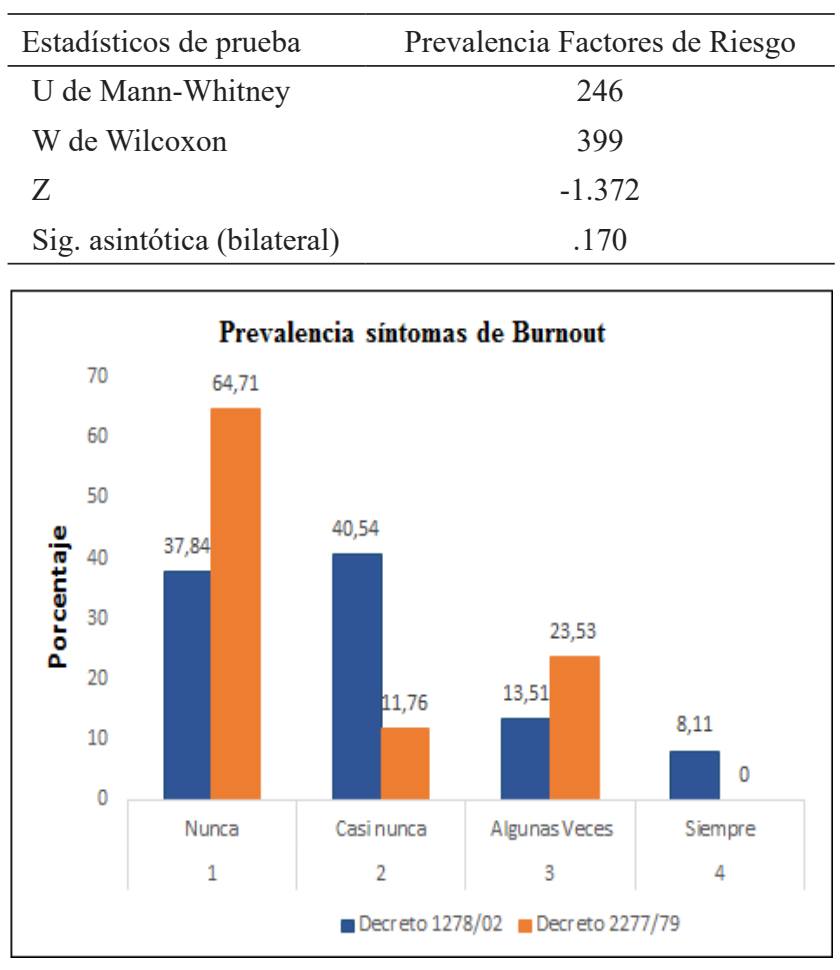

Figura 4. Principales síntomas que generan en el síndrome Burnout en docentes de los decretos 1278 y 2277 de la I. E. Técnica San José, de Fresno (Tolima).

El índice U de Mann-Withney de 246 con un $p=.170(17 \%$ de error) permite rechazar la hipótesis de diferencia entre las observaciones de ambos grupos sobre la presencia de síntomas de Burnout, esto se puede constatar en la Figura 4.

\section{Discusión y Conclusiones}

El Burnout es un trastorno que afecta principalmente a profesionales que tienen contacto directo con personas, en especial aquellos con altas tasas de entrega y compromiso laboral como es el caso de docentes, lo que puede repercutir en un deterioro en la salud (Gutiérrez et al, 2006; Ingaruca, 2017; Redó, 2017).

Diversos estudios han demostrado la prevalencia del síndrome en profesores (Aldrete et al. 2003; Bórquez, 2004), algunos de los cuales han hallado niveles altos de Burnout en docentes de secundaria en el país (Díaz et al., 2010; CorreaCorrea et al., 2010; Padilla et al. 2009; Yepes, 2006).

En el presente estudio se buscó determinar los factores de riesgo y síntomas de Burnout en docentes de secundaria de un colegio de Colombia. Se encontró que el $45.9 \%$ de docentes 1278 y un $41.2 \%$ del 2277 presentaron una susceptibilidad media, mientras que $29.7 \%$ del 1278 y un $29.4 \%$ del 2277 moderado. De manera, que se establece que la mayoría de los docentes de la institución estudiada presentan una susceptibilidad media, lo que concuerda con diversas investigaciones anteriormente citadas, pero se resalta que hay mayor prevalencia en docentes del decreto 1278 .

Galdeano, Godoy \& Cruz (2007) en su indagación sobre factores de riesgo psicosocial en profesores de secundaria, encontraron que el estrés laboral ocupa un segundo lugar, por detrás 
de las enfermedades osteomusculares. A su vez hallaron que éste afecta una alta proporción del profesorado de secundaria y que entre los factores que se le atribuyen al mismo se ha sugerido que las reformas educativas, alto número de estudiantes en una misma institución, la desmotivación y déficit de atención del alumnado, la presión social y la falta de apoyo de compañeros y equipo directivo son los principales atenuantes.

En el presente estudio se encontró que los principales factores que inciden en Burnout en la institución estudiada son: trabajar horas extras, falta de espacios de comunicación con los jefes, un salario inadecuado para satisfacer sus necesidades y falta de apoyo de los jefes frente a dificultades con padres de familia. Concordando con la pesquisa de Galdeano et al. (2007) y de Díaz et al. (2010), en cuanto a la desmotivación y supervisión del equipo directivo y organización, lo que se señala que estos inciden directamente en la presencia del síndrome.

De igual manera Aldrete et al. (2003), han considerado que los factores laborales que provocan mayor estrés son: volumen de trabajo, factores relacionados con los alumnos, salario inadecuado, déficit de recursos materiales, escasez de equipos y facilidades para el trabajo. Lo que coincide con este análisis, pero cabe resaltar que este estudio, señala mayor prevalencia en docentes 1278, posiblemente debido a que estos son remunerados en menor medida y presentan mayores factores de estrés, además de tener menor apoyo de los superiores para ejercer su ejercicio docente con respecto a los 2277. A su vez esto coincide con la postulación de Galdeano et al. (2007) en relación a que las reformas educativas tienen una alta prevalencia en la presencia del síndrome.

Como se resalta al inicio del artículo, este síndrome se está convirtiendo en un serio problema no sólo para el colectivo docente, sino también para el sistema educativo en general, por las consecuencias directas con la calidad de la enseñanza y sus efectos negativos crecientes en bajas laborales, rotación, absentismo y decrecimiento en la productividad de la enseñanza (Doménech, 1995; Extremera et al, 2003). En correspondencia con León et al., (2008) la salud mental de los docentes es un problema actual que debe ser considerado y que puede estar asociado con el síndrome de Burnout.

La ruta metodológica usada en el presente estudio demostró que hay diferencias entre la prevalencia del síndrome de Burnout entre docentes de ambos estatutos, así mismo al indagar los principales factores causales, se determinaron diferencias significativas, de manera que una de las conclusiones primordiales, es que los dos principales factores que determinan la mayor prevalencia de Burnout en docentes 1278 frente a los 2277 en la Institución Educativa. son: la comunicación con superiores y un salario insuficiente para satisfacer las necesidades de los docentes.

De manera que se señala como uno de los problemas transcendentales de la actualidad a que se ven enfrentados los docentes nuevos (decreto 1278), y que se hace evidente desde la década pasada, es la dificultad de la comunicación con superiores, esto en razón a que desde la creación del decreto 1278 y la división subsecuente de los docentes bajo diferentes rangos de calificación y salarios. Estos se han visto sometidos a tensiones de jerarquía, como las evaluaciones periódicas $y$ anuales que hacen sus superiores de acuerdo a dicho nombramiento, lo que ha generado una presión significativa y que podría ser una de las razones de la presencia del síndrome en docentes 1278 frente a los 2277.

Así mismo, el salario insuficiente para satisfacer las necesidades de los docentes es otro ítem importante a tener en cuenta, que manifiesta diferencias entre uno y otro estatuto, en razón a la baja motivación y desgaste por parte de los docentes, tal como se ha evidenciado en otros estudios, estos dos problemas laborales que 
muestran marcadas diferencias significativas entre uno y otro estatuto son factores que se hacen evidentes en las discusiones que se presentan con regularidad en instituciones educativas.

Con respecto al salario insuficiente, Vázquez, Esperón \& González (2013) han señalado que la remuneración es una de las variables principales involucradas en la aparición del Síndrome de Burnout en enfermeras y postulan que las personas con salarios más bajos son más afectados frente a los mejores remunerados, tal como sucede entre los decretos 1278 y 2277 respectivamente. Concluye que a menor salario mayor frecuencia del síndrome, asimismo que jornadas laborales generaban mayor prevalencia, lo que concuerda con lo encontrado en esté estudio.

Por su parte, Caballero et al. (2009) al estudiar los factores de riesgo psicosocial de la organización universitaria, hallaron que un tercio de la muestra tenía preocupaciones profesionales y percepciones de falta de reconocimiento de la Institución. Cuandohabía mayores preocupaciones por el bajo salario, mayor afectación de la imagen pública y de percepción de falta de apoyo organizacional.

En síntesis, las correlaciones encontradas, ponen de manifiesto que el síndrome se asocia con variables del contexto organizacional, lo cual apoya la postura de los diferentes modelos teóricos que reconocen la naturaleza psicosocial de Burnout y concuerdan ampliamente con nuestra investigación.

En cuanto a las estrategias y teorías para abordar este problema, Salanova, Schaufeli, Llorens, Peiró y Grau (2000) señalan que el Engagement se relaciona con la disponibilidad de recursos laborales, favorece un mayor compromiso organizacional (p.e., Satisfacción y Entusiasmo laboral), y ayuda a controlar la prevalencia del Burnout en los trabajadores. En definitiva, los estudios han mostrado vínculos positivos entre indicadores de bienestar laboral (Durán, Extremera, Montalbán \& Rey, 2005).

Para Fernández \& Paravic (2003) “trabajar en ambientes laborales más confortables, seguros y agradables, con políticas de remuneraciones y de promociones y ascensos justas y equitativas, todo lo cual influirá en la satisfacción laboral". De manera que se propone que una acción a desarrollar encaminada a disminuir la prevalencia de Burnout en docentes, especialmente del decreto 1278 , es contemplar: estrategias personales cognitivoconductuales con carácter auto evaluativo que reduzcan o eliminen el estrés, desarrollar habilidades de comunicación e impulsar el apoyo social entre los profesionales, para disminuir los estresores del entorno laboral, tal como lo señalan Araujo y Solís (2017).

Por tanto, adecuadas habilidades de comunicación contribuyen para que el profesional se sienta más seguro, competente y fomente una adecuada relación interpersonal, reduciendo el estrés laboral (Rivera, Martínez, Lizárraga \& Rojas, 2011). Anivel organizacional, es importante eliminar o disminuir los estresores del entorno organizacional que dan lugar al desarrollo del síndrome. Lo cual, según Rivera et al. (2011), se logra modificando el ambiente físico, la estructura organizacional, las funciones de los puestos, las políticas de administración de recursos humanos, entre otros, que tienen como propósito generar estructuras horizontales y descentralizadas en la toma de decisiones.

Lo que permite brindar mayor independencia y autonomía, así como flexibilidad horaria, sueldos competitivos, ente otros. Finalmente se recomienda seguir indagando al respecto y desarrollar investigaciones que relacionen con otros factores que pueden intervenir en la generación del síndrome. De manera que es importante de acuerdo a lo evidenciado en este proyecto que se mejoren y se facilite la comunicación con los superiores así como generar un salario suficiente para los docentes del 1278 . 


\section{Referencias}

Aldrete Rodríguez, M., \& Pando Moreno, M., \& Aranda Beltrán, C., \& Balcázar Partida, N. (2003). Síndrome de Burnout en maestros de educación básica, nivel primaria de Guadalajara. Investigación en Salud, V (1). Recuperado de https://www.redalyc. org/html/142/14200103/

Aragüez Valenzuela, L. (2017). El impacto de las tecnologías de la información y de la comunicación en la salud de los trabajadores: el tecnoestrés. Revista Internacional de la Protección Social, 2 (2), 169-190.

Araujo Lugo, M. V., \& Solís Marín, K. E. (2017). Habilidades de comunicación efectiva $y$ su relación, con la presencia de síndrome de Burnout en médicos postgradistas de áreas clínicas y quirúrgicas de la Pontificia Universidad Católica del Ecuador, en el período 2012-2016. (Tesis de Especialización). Pontificia Universidad Católica del Ecuador. Ecuador.

Bórquez B., S. (2004). Burnout, o estrés circular en profesores. Pharos, 11(1), 23-34.

Caballero Domínguez, C., González Gutiérrez, O., Mercado Anguila, D., Llanos Barrios, C., Bermejo Barrero, Y., \& Vergel Medrano, C. (2009). Prevalencia del Síndrome de Burnout y su correlación con factores psicosociales en docentes de una institución universitaria privada de la ciudad de Barranquilla. Psicogente, 12(21), 142-157.

Campoy, T. J. (2018). Metodología de la investigación Científica. Manual para la elaboración de Tesis y Trabajos de Investigación. Asunción, Paraguay: Marben.
Cifuentes Cubillos, C. (2013). Estatuto de Profesionalización Docente en Colombia Análisis de los dos estatutos vigentes (Decreto 2277 de 1979 y Decreto 1278 de 2002). Diferencias en la labor docente. (Tesis de maestría) Universidad Nacional de Colombia. Colombia.

Correa-Correa, Z., Muñoz-Zambrano, I., \& Chaparro, A.F. (2010). Síndrome de Burnout en docentes de dos universidades de Popayán, Colombia. Revista de salud pública, 12(4), 589-598.

Díaz Bambula, F., López Sánchez, A., \& Varela Arévalo, M. (2010). Factores asociados al síndrome de Burnout en docentes de colegios de la ciudad de Cali, Colombia. Universitas Psychologica, 11(1), 217-227.

Doménech Delgado, B. (1995). Introducción al síndrome "Burnout" en profesores y maestros y su abordaje terapéutico. Psicología Educativa, 1(1), 63-78.

Durán, M. A, Extremera, N., Montalbán, F. M., \& Rey, L. (2005). Engagement y Burnout en el ámbito docente: Análisis de sus relaciones con la satisfacción laboral y vital en una muestra de profesores. Revista de Psicología del Trabajo y de las Organizaciones, 21(1-2), 145-158.

Extremera, N., Fernández-Berrocal, P., \& Durán Durán, A. (2003). Inteligencia emocional y Burnout en profesores. Encuentros en psicología social, 1(5), 260-265.

Fernández, B., \& Paravic, T. (2003). Nivel de satisfacción laboral en enfermeras de hospitales públicos y privados de la provincia de Concepción, Chile. Ciencia y enfermería, 9(2), 57-66.

Galdeano, H., Godoy, P., \& Cruz, I. (2007). Factores de riesgo psicosocial en 
profesores de educación secundaria. Arch Prev Riesgos Labor, 10(4), 174-180.

Guevara, C., Henao, D., \& Herrera, J. (2004). Síndrome de desgaste profesional en médicos internos y residentes. Colombia Médica, 35(4), 173-178.

Gutiérrez, A., Celis, M.A., Moreno, S., Farías, F., \& Suárez (2006). Síndrome de Burnout. Archivos de Neurociencias, 11(4), 305309.

Hermosa, A. (2006). Satisfacción laboral y síndrome de "Burnout" en profesores de educación primaria y secundaria. Revista Colombiana de Psicología, 15, 81-89.

Ingaruca, B.B. (2017). Nivel de síndrome de Burnout en docentes de una Institución Educativa Pública del Distrito de Santiago de Surco-2017. Lima, Perú. Universidad Inca Garcilaso de la Vega. p. 73.

Knight-Wegenstein, A.G. (1973). Die arbeitszeit der Lehrer in der Bundesrepublik Deutschland. Zürich. Band I: Analyse, Band II: Katalog für Zeitaufwand. Knight \& Wegenstein AG, Zürich.

León, S., Matsui, O., Aranda, C. \& León, M. (2008). Factores psicosociales y Síndrome de Burnout en docentes de educación básica del estado de Jalisco. Estrés y salud mental en el trabajo. 2 Foro de las Américas en Investigación sobre factores Psicosociales. $10 \mathrm{p}$.

López, C. (2004). Síndrome de Burnout. Revista mexicana de anestesiología, 27(1), 13133.

Macia, M. B. (2009). La profesionalización docente en Colombia. Revista colombiana de sociología, 32(2), 111-132.
Maslach, C. (1982). Burnout: the cost of caring. New York: Prentice-Hall Press.

Maslach, C. y Jackson, S. E. (1981). The measurement of experienced Burnout. Journal of Occupational Behaviour, 2, 99113.

Medina, L. A. (2015). Innovación y gestión del conocimiento; síndromes laborales necesarios de extinguir. RIDE Revista Iberoamericana para la Investigación y el Desarrollo Educativo, 6(11).

Méndez, V. J. (2004). Estrés laboral o síndrome de "Burnout". Acta Pediátrica de México, 25(5), 299-302.

Moriana Elvira, J.A., \& Herruzo Cabrera, J. (2004). Estrés y Burnout en profesores. International journal of clinical and health psychology, 4(3), 597-621.

Ortega, E. M. \& Giménez, L. (2013). Síndrome de Burnout: una aplicación a profesorado de Educación secundaria obligatoria, bachillerato y ciclos formativos. Master Universitario de profesorado en educación secundaria obligatoria y bachillerato, formación profesional y enseñanza de idiomas. Universidad de Almería, España; $46 \mathrm{p}$.

Ortega, C., \& López, F. (2004). El Burnout o síndrome de estar quemado en los profesionales sanitarios: revisión y perspectivas. International Journal of Clinical and Health Psychology, 4(1), 137-60.

Padilla, A. C., Gómez, C., Rodríguez, V., Dávila, M., Avella, C. B., Caballero, A., Vives, N., Mora, C., Márquez, G., Prieto, Y., Sandoval, N., Cotes, Z., \& Hernández, S. (2009). Prevalencia y características del síndrome de agotamiento profesional 
(SAP) en docentes de tres colegios públicos de Bogotá (Colombia). Revista colombiana de psiquiatría, 38(1).

Palmer, Y., Gómez-Vera, A., Cabrera-Pivaral, C., Prince-Vélez, R., \& Searcy, R. (2005). Factores de riesgo organizacionales asociados al síndrome de Burnout en médicos anestesiólogos. Salud Mental, 28(1), 82-91.

Pérez, A. M. (2010). El síndrome de Burnout. Evolución conceptual y estado actual de la cuestión. Vivat Academia, (112), 42-80.

Ponce, C. R., Bulnes, M. S., Aliaga, J. R., Atalaya, M. C. \& Huertas, R. E. (2005). El síndrome del "quemado" por estrés laboral asistencial en grupos de docentes universitarios. Revista IIPSI, 8(2), 87-112.

Porras, N. P. (2017). Análisis de la relación entre la salud mental y el malestar humano en el trabajo. Equidad \& Desarrollo, (29, suplemento), 161-178. doi: 10.19052/ ed. 4173

Redó, N. A. (2017). El Síndrome de Burnout en los docentes. Electronic Journal of Research in Education Psychology, 7(18), 829-848.

Restrepo, N. C., Colorado, G. O. \& Cabrera, G. A. (2006). Desgaste Emocional en Docentes Oficiales de Medellín: Colombia, Rev. Salud pública, 8(1), 63-73.

Rivera, B. R. G., Martínez, I. A. M., Lizárraga, R. \& Rojas, A. A. G. (2011). Estudio exploratorio del Síndrome de Burnout en una Institución de Educación Superior Mexicana usando la escala de Maslach. Revista Internacional Administración \& Finanzas, 4(2), 67-81.

Rojas, M. L., Zapata, J. A., \& Grisales, H. (2009). Síndrome de Burnout y satisfacción laboral en docentes de una institución de educación superior, Medellín, 2008. Revista Facultad Nacional de Salud Pública, 27(2), 198-210.

Salanova, M., Schaufeli, W., Llorens, S., Peiró, J. \& Grau, R. (2000). Desde el "Burnout" al "Engagement" ¿Una nueva perspectiva? Revista de Psicología del Trabajo y de las organizaciones, 16(2), 117-134.

Saquero, A.; Jaime, G.L.; Ortín, F. (2018). Relación entre el síndrome de Burnout, niveles de optimismo, edad y experiencia profesional en entrenadoras y entrenadores de gimnasia rítmica. Journal of Sport and Health Research, 10(1):79-90.

Sauter, S. L., Murphy, L. R., Hurrell, J. J., \& Levi, L. (2001). Factores psicosociales y de organización. Capítulo 34. Enciclopedia de salud y seguridad en el trabajo. $3^{\text {ra }}$ Ed. España: Organización Internacional del Trabajo y Ministerio de Trabajo y Asuntos Sociales.

Yépez, M. \& Soracá, A. (2006). Síndrome de Burnout Asociado con Factores de Riesgos Psicosociales en docentes oficiales del municipio de San Andrés de Sotavento, Córdoba. (Especialistas en salud ocupacional). Medellín, Antioquia: Facultad Nacional de Salud Pública.

Vázquez, C., Esperón, R., \& González, M. (2013). Síndrome de Burnout en cuidadores de adultos mayores que trabajan en casas de asistencia social. Revista de Investigación Clínica, 65(6); 510-514.

Viloria, H., \& Paredes, M. (2002). Estudio del síndrome de Burnout o desgaste profesional en los profesores de la Universidad de los Andes. Educere, 6(17), 29-36. 